\title{
Hybridization and antibiotic synergism as a tool for reducing the cytotoxicity of antimicrobial peptides
}

This article was published in the following Dove Press journal:

Infection and Drug Resistance

\author{
Ammar Almaaytah' \\ Mohammed T Qaoud \\ Ahmad Abualhaijaa ${ }^{2}$ \\ Qosay Al-Balas ${ }^{3}$ \\ Karem H Alzoubi ${ }^{4}$ \\ 'Department of Pharmaceutical \\ Technology, Faculty of Pharmacy, \\ Jordan University of Science and \\ Technology, Irbid, Jordan; ${ }^{2}$ Department \\ of Applied Biological Sciences, Faculty \\ of Science and Arts, Jordan University \\ of Science and Technology, Irbid, \\ Jordan; ${ }^{3}$ Department of Medicinal \\ Chemistry, Faculty of Pharmacy, \\ Jordan University of Science and \\ Technology, Irbid, Jordan; ${ }^{4}$ Department \\ Clinical Pharmacy, Faculty of \\ Pharmacy, Jordan University of \\ Science and Technology, Irbid, Jordan
}

Introduction: As the development of new antimicrobial agents faces a historical decline, the issue of bacterial drug resistance has become a serious dilemma that threatens the human population worldwide. Antimicrobial peptides (AMPs) represent an attractive and a promising class of antimicrobial agents.

Aim: The hybridization of AMPs aimed at merging two individual active fragments of native peptides to generate a new AMP with altered physicochemical properties that translate into an enhanced safety profile.

Materials and methods: In this study, we have rationally designed a new hybrid peptide via combining two individual $\alpha$-helical fragments of both BMAP-27 and OP-145. The resultant peptide, was evaluated for its antimicrobial and antibiofilm activity against a range of microbial strains. The resultant peptide was also evaluated for its toxicity against mammalian cells using hemolytic and anti proliferative assays.

Results: The antimicrobial activity of $\mathrm{H} 4$ revealed that the peptide is displaying a broad spectrum of activity against both Gram-positive and Gram-negative bacteria including standard and multidrug-resistant bacterial strains in the range of $2.5-25 \mu \mathrm{M}$. The new hybrid peptide displayed potent activity in eradicating biofilm-forming cells, and the reported minimum biofilm eradication concentrations were equal to the minimum inhibitory concentration values reported for planktonic cells. Additionally, $\mathrm{H} 4$ exhibited reduced toxicity profiles against eukaryotic cells. Combining $\mathrm{H} 4$ peptide with conventional antibiotics has led to a dramatic enhancement of the antimicrobial activity of both agents with synergistic or additive outcomes.

Conclusion: Overall, this study indicates the success of both the hybridization and synergism strategy in developing AMPs as potential antimicrobial therapeutics with reduced toxicity profiles that could be efficiently employed to eradicate resistant bacterial strains and enhance the selectivity and toxicity profiles of native AMPs.

Keywords: antimicrobial peptides, peptide hybridization, antibiotic synergism, biofilms, antimicrobial resistance

\section{Introduction}

The unprecedented use of antibiotics in the recent decades for the treatment of human infections has contributed significantly to the emergence of a high proportion of bacterial pathogens that are displaying resistance to multiple antibacterial agents. ${ }^{1}$ This significant rise in antimicrobial resistance has been accompanied by a sharp decrease in the development of novel groups of antimicrobial agents to tackle this worldwide problem. ${ }^{2}$ As the number of antibiotics approved by the US Food and Drug Administration for the treatment of systemic infections has declined by $90 \%$ during the last 30 years, there have been several global initiatives to reinvigorate the antibiotic pipelines
Correspondence: Ammar Almaaytah Department of Pharmaceutical Technology, Faculty of Pharmacy, Jordan University of Science and Technology, P.O Box 3030, Irbid 22110, Jordan Tel +962777658820

Fax +96227201075

Email amalmaaytah@just.edu.jo 
and prevent the human population from reaching the postantibiotic era. ${ }^{3}$ Antimicrobial peptides (AMPs) have been studied extensively as an alternative group of antimicrobial agents that could represent a novel class of molecules capable of combating bacterial pathogens and combating microbial resistance. ${ }^{4}$ Several AMPs have been shown to be capable of eradicating several microorganisms including bacteria, fungi, and viruses. ${ }^{5}$ As these molecules display a huge diversity in their structure, a broad spectrum of activity, and a low propensity to induce microbial resistance, several attempts focused on the development of these agents into clinically successful therapeutics. ${ }^{6}$ Despite the attractive feature that AMPs possess, their development into effective antimicrobials has been hampered by several obstacles including high mammalian cell cytotoxicity due to a lack of target selectivity in addition to high manufacturing costs and low metabolic stability. ${ }^{7}$

Several reports have shown that BMAP-27 exhibits significant antimicrobial activity against wild-type and resistant strains of bacteria. ${ }^{8}$ In addition, the toxicity profile of BMAP-27 against mammalian cells was significantly high, limiting any possibility for the advancement of the molecule toward clinical development. ${ }^{9} \mathrm{OP}-145$ is a human cathelicidin LL-37-derived AMP with potent antimicrobial activities and relatively tolerable selectivity. It has been proven as a safe and successful treatment option for chronic otitis media in a clinical phase I/II trial. ${ }^{10}$

In the present study, we have rationally designed a novel hybrid AMP aiming at retaining the potent antimicrobial activity of both BMAP-27 and OP-145 while reducing the toxicity profile of the product peptide against mammalian cells. In order to enhance its selectivity index and reduce its overall toxicity, ${ }^{11}$ the designed peptide was combined with conventional antibiotics to assess the synergistic outcome of these combinations. The design strategy focused on combining individual $\alpha$-helical fragments from both BMAP-27 and OP-145 to generate a novel hybrid peptide with an enhanced safety profile and a capability of displaying potent synergistic activities when combined with conventional antibiotics. Named H4, the antimicrobial and antibiofilm activities of $\mathrm{H} 4$ were evaluated against a range of Gram-positive and Gramnegative bacterial strains in addition to multidrug-resistant (MDR) bacteria (MDRB). The synergistic effects of combining $\mathrm{H} 4$ with conventional antibiotics were also assessed for the purpose of identifying any synergistic patterns that would lower the effective minimum inhibitory concentration (MIC) of the peptide and consequently decrease the toxicity of the peptide. H4's toxicity was also evaluated using hemolytic assays in addition to antiproliferative studies against mammalian cells.

\section{Materials and methods Peptide design and analysis}

The computational models for the prediction of the structural properties of the designed peptide were based on computational software as reported previously. ${ }^{12}$ The $\alpha$-helical fragments from both BMAP-27 and OP-145 employed in the design of the hybrid peptide $\mathrm{H} 4$ were identified through the protein sequence analysis tool of Hierarchical Neural Network in Network Protein Sequence (NPS HNN) analysis. ${ }^{13}$ The percentage of the overall $\alpha$-helical content of the designed peptide was calculated using the same software. The HydroMCalc software was employed for calculating the mean hydrophobicity $(<\mathrm{H}>)$ and the hydrophobic moment $(<\mu \mathrm{H}>) \cdot{ }^{14}$ Innovagen's peptide calculator was used for calculating the water solubility, net charge at neutral $\mathrm{pH}$, molecular weight, and isoelectric point of the parent and hybrid peptides. ${ }^{15}$ Finally, the ProtParam software from the ExPASy server was employed for the evaluation of the physicochemical parameters of $\mathrm{H} 4$.

\section{Peptide synthesis and purification}

H4 was synthesized using solid-phase fluorenylmethyloxycarbonyl (Fmoc) chemistry (GL Biochem, Shanghai, China). The purity ( $>95 \%$ ) of the peptide was evaluated by employing high-performance liquid chromatography (HPLC). Electron spray ionization-mass spectrometry (ESI-MS) was used to confirm the identity of the synthetic $\mathrm{H} 4$ peptide.

\section{Molecular modeling and in silico analysis of $\mathrm{H} 4$}

The physicochemical parameters of $\mathrm{H} 4$ were analyzed using the ProtParam software. ${ }^{16}$ The HHpred (HHsearch 2.0) software was employed for identifying the optimal template for homology modeling of H4. ${ }^{17}$ Packing and solvent exposure characteristics were analyzed using the PROSA software. ${ }^{13}$ The RAMPAGE: Assessment of the Ramachandran Plot software was also used for the three-dimensional structure validation. ${ }^{18}$ The final model was visualized using Accelrys ${ }^{\circledR}$ Discovery Studio software.

\section{Microorganisms}

The microorganisms used in the study were acquired from the American Type Culture Collection (ATCC; Manassas, VA, USA) as the eight control bacterial strains were employed in 
the study for the evaluation of the antimicrobial activity of the hybrid peptide H4. The microorganisms include Staphylococcus aureus (ATCC 29213), Staphylococcus epidermidis (ATCC 12228), Enterococcus faecalis (ATCC 19433), Klebsiella pneumoniae (ATCC 13883), Escherichia coli (ATCC 25922), Pseudomonas aeruginosa (ATCC 27853), P. aeruginosa (ATCC 47083), and Acinetobacter baumannii (ATCC 19606). In addition, the antimicrobial activity of H4 was also evaluated against seven standard resistant strains and clinical isolates of bacteria including Gram-positive MDR S. aureus (ATCC 43300, 33591, and BAA41); two strains of MDR Enterococcus faecium (ATCC BAA2356 and BAA2316); and two Gram-negative bacterial strains including MDR P. aeruginosa (ATCC BAA2114), and MDR E. coli (ATCC BAA2452).

\section{Antibiotics}

Conventional antibiotics levofloxacin, chloramphenicol, rifampicin, and ampicillin were obtained from Sigma-Aldrich (St. Louis, MO, USA), and erythromycin was obtained from Sigma-Aldrich. According to the manufacturer's recommendations, the antibiotic powders and stock solution preparations were stored at the optimum temperature for each antibiotic.

\section{Bacterial susceptibility assay}

The standard microbroth dilution method as outlined by the Clinical and Laboratory Standards Institute guidelines was employed for the determination of the minimum inhibitory concentrations (MICs) of the hybrid peptide H4 against the bacterial strains mentioned previously. ${ }^{18,19}$

Briefly, Muller-Hinton Broth (MHB; Oxoid Ltd., Basingstoke, UK) was used as a growth medium for overnight bacterial cell growth followed by dilution to $10^{6} \mathrm{CFU} / \mathrm{mL}$ employing the same medium prior to the experiment. The $\mathrm{H} 4$ hybrid peptide was serially diluted in MHB at a final volume of $50 \mu \mathrm{L} /$ well using 96 -well plates. The concentration range of $\mathrm{H} 4$ for the bacterial susceptibility assay was $0.5-25 \mu \mathrm{M}$. The final concentration of the bacterial cells, after dilution, was $5 \times 10^{5} \mathrm{CFU} / \mathrm{mL}$. Plates were incubated for 18 hours, at $37^{\circ} \mathrm{C}$, in a humidified atmosphere. Following this, bacterial growth was determined by measuring optical density (OD) at $\lambda=570 \mathrm{~nm}$ using an enzyme-linked immunosorbent assay (ELISA) plate reader (Epoch ${ }^{\mathrm{TM}}$; BioTek, Winooski, VT, USA). The growth of bacteria was determined by means of measuring OD by an ELISA plate reader. The minimum bactericidal concentration (MBC) was identified by inoculating $10 \mu \mathrm{L}$ of the each well on agar and grown at $37^{\circ} \mathrm{C}$ for 24 or 48 hours. All MIC and $\mathrm{MBC}$ determinations were made in triplicate.

\section{Synergistic evaluation of the peptide and antibiotics in combinations}

The checkerboard technique as described previously was employed for the determination of the synergistic effect of $\mathrm{H} 4$ and the antibiotics in combination against wild-type and resistant strains of bacteria. ${ }^{20}$ Serially diluted concentrations of each antibiotic and the $\mathrm{H} 4$ peptide were prepared in the range of $0.005-100 \mu \mathrm{M}$. The MIC and MBC of each individual combination were determined as described in the "Bacterial susceptibility assay" section. All MIC and MBC determinations of all assays were made in triplicate.

\section{Fractional inhibitory concentration (FIC)}

The degree and the assessment of the synergy between two antimicrobial agents were determined and expressed by using the FIC index that is defined as the inhibitory concentration of the antimicrobial combination divided by that of the single antimicrobial component. The following equation represents the method for determining the FIC index:

$$
\begin{array}{r}
\text { FIC index }=\frac{\text { MIC of drug X in combination }}{\text { MIC of drug X alone }}+ \\
\frac{\text { MIC of drug Y in combination }}{\text { MIC of drug Y alone }}
\end{array}
$$

The FIC indices were interpreted as follows: $\leq 0.5$ : synergistic activity, 0.5-1: additive activity, 1-4: indifferent, >4: antagonism. Interpretation and assessment of the FIC indices were performed according to the broth microdilution checkerboard technique. ${ }^{21,22}$

\section{Antibiofilm activity of $\mathrm{H} 4$ against resistant strains of Gram-positive and Gram- negative bacteria}

The Calgary Biofilm Device (Innovatech, Edmonton, AB, Canada) was employed for biofilm formation as described previously. ${ }^{23}$ Briefly and according to the manufacturer's instructions, both bacterial strains of resistant $P$. aeruginosa (ATCC BAA2114) and S. aureus (ATCC 43300) were incubated in $\mathrm{MHB}$ for 20 hours at $37^{\circ} \mathrm{C}$, followed by dilution of the bacterial concentration of both species to $10^{7} \mathrm{CFU} /$ $\mathrm{mL} ; 150 \mu \mathrm{L}$ from each bacterial strain was inoculated with 96 pegs-lids found on the device to allow biofilm growth formation. After biofilm formation, the pegs were washed 
with phosphate buffered saline (PBS), and each peg-lid was then transferred into a "challenge 96-well microtiter plate" with different concentrations of $\mathrm{H} 4$ and incubated for 4 hours at $37^{\circ} \mathrm{C}$. The minimum biofilm eradication concentration (MBEC) is defined as the minimum concentration needed to inhibit the regrowth of biofilms after 4 hours of peptide treatment using an ELISA plate reader at $\lambda=550 \mathrm{~nm}$. In addition, the biofilms were assessed for their $\mathrm{MBC}$, as this parameter is defined as the lowest concentration that can eradicate $3 \log _{10}$ of the viable microorganisms in a biofilm (99.9\% killing) after 2 hours of incubation using the colony count method.

\section{Hemolytic assay}

The toxicity of H4 against human erythrocytes was determined using the hemolytic assay in order to assess the degree of hemolysis induced by the peptide and as described previously. ${ }^{24}$ Briefly, a 4\% suspension of human erythrocytes (Zen-Bio Inc., Research Triangle Park, NC, USA) in 0.9\% sodium chloride $(\mathrm{NaCl})$ was prepared and incubated with different concentrations of $\mathrm{H} 4$ at $37^{\circ} \mathrm{C}$ for 1 hour. Triton $\mathrm{X}-100$ was used as a positive control in order to induce $100 \%$ hemolysis, while erythrocytes lacking the peptide were employed as negative controls. The percent hemolysis was calculated using the following equation: hemolysis = $(\mathrm{A}-\mathrm{A} 0) /(\mathrm{AX}-\mathrm{A} 0)^{*} 100$, where $\mathrm{A}$ is $\mathrm{OD} 570 \mathrm{~nm}$ with the peptide solution, $\mathrm{A} 0$ is $\mathrm{OD} 570 \mathrm{~nm}$ in $\mathrm{NaCl}$, and $\mathrm{AX}$ is OD $570 \mathrm{~nm}$ with $0.1 \%$ Triton $\mathrm{X}-100$.

\section{Mammalian cytotoxicity assay}

For determining the cytotoxicity of $\mathrm{H} 4$ against mammalian cells, two cell lines, HEK293 and Vero, were acquired from the ATCC and were employed as model in vitro cells for assessing the peptide cytotoxicity against mammalian cells. Each cell line used was seeded at a density of $5 \times 10^{3}$ cells per well on a 96-well plate. The cells were exposed to different concentrations of $\mathrm{H} 4$ including the medium alone as a negative control. They were treated with various concentrations of H4 or with medium alone. Following this, the plates were incubated for 24 hours, after which $20 \mu \mathrm{L}$ of $5 \mathrm{mg} /$ mL 3-(4,5-dimethylthiazol-2-yl)-2,5-diphenyltetrazolium bromide (MTT) solution was added to each well and the plates were incubated again for 3 hours. The growth medium was later removed using a 1-mL syringe fitted with a needle, and $200 \mu \mathrm{L}$ of dimethyl sulfoxide (DMSO) was added to each well and mixed vigorously to dissolve the formazan crystals that had developed. The absorbance was measured by using an absorbance microplate reader at $550 \mathrm{~nm}$.

\section{Results}

\section{Peptide synthesis and purification}

Conventional solid-phase Fmoc chemistry was employed for the synthesis of H4 (GL Biochem). Reverse-phase HPLC was employed to determine the purity of the peptide, and the chromatogram indicated that the peptide purity was $>95 \%$ and in accordance with standard purity recommendations for peptide synthesis required for in vitro studies (Figure 1). The identity of the peptide was determined by ESI-MS as the synthetic replicate of $\mathrm{H} 4$ showing major peaks in the +4 , $+5,+6$, and +7 charge states of $910.27,728.38,607.16$, and 520.68 Da, respectively (Figure 2).

\section{Design of H4 hybrid peptide}

The strategy employed for the design of H4 depended on identifying the $\alpha$-helical fragments within the primary sequence of the parent peptides employed in this study in order to combine these fragments to generate a hybrid peptide with enhanced and stabilized physicochemical properties that could be translated in vitro in retaining the antimicrobial activity of the parent peptides with an enhanced toxicity profile. Accordingly, both primary peptide sequences of BMAP-27 and OP-145 were evaluated for the percentage helicity employing the NPS HNN secondary structure prediction software and for the identification of the $\alpha$-helical fragments within each peptide (Table 1). The results displayed that the percentage helicity for BMAP-27 was $73.08 \%$, while OP-145 exhibited a percentage helicity of $91.8 \%$. Both peptides exhibited three potential $\alpha$-helical fragments suitable for hybridization. These fragments were employed for creating several combinations of hybrid peptides that were subsequently evaluated for their overall percentage helicity, as well as other physicochemical parameters such as net charge, hydrophobicity, and hydrophobic moment. The resultant peptides were also screened for their antimicrobial activity. Using this approach, five novel hybrid peptides were evaluated for potential antimicrobial activity with only one hybrid peptide named $\mathrm{H} 4$ displayed promising antimicrobial activity in the initial screening assays (data not shown). H4 consists of an N-terminal fragment obtained from residues 9-26 of BMAP-27 and a C-terminal fragment from residues 1-16 of OP-145 (Table 2). H4 is composed of 29 amino acid residues and displays a total percentage helicity of $90 \%$, a net charge of +6 , a hydrophobicity of 0.272 , and a hydrophobic moment of 0.64 (Table 2). Compared with the parent peptides, $\mathrm{H} 4$ displays an enhanced helicity profile in addition to an optimized net charge and a hydrophobicity index. 


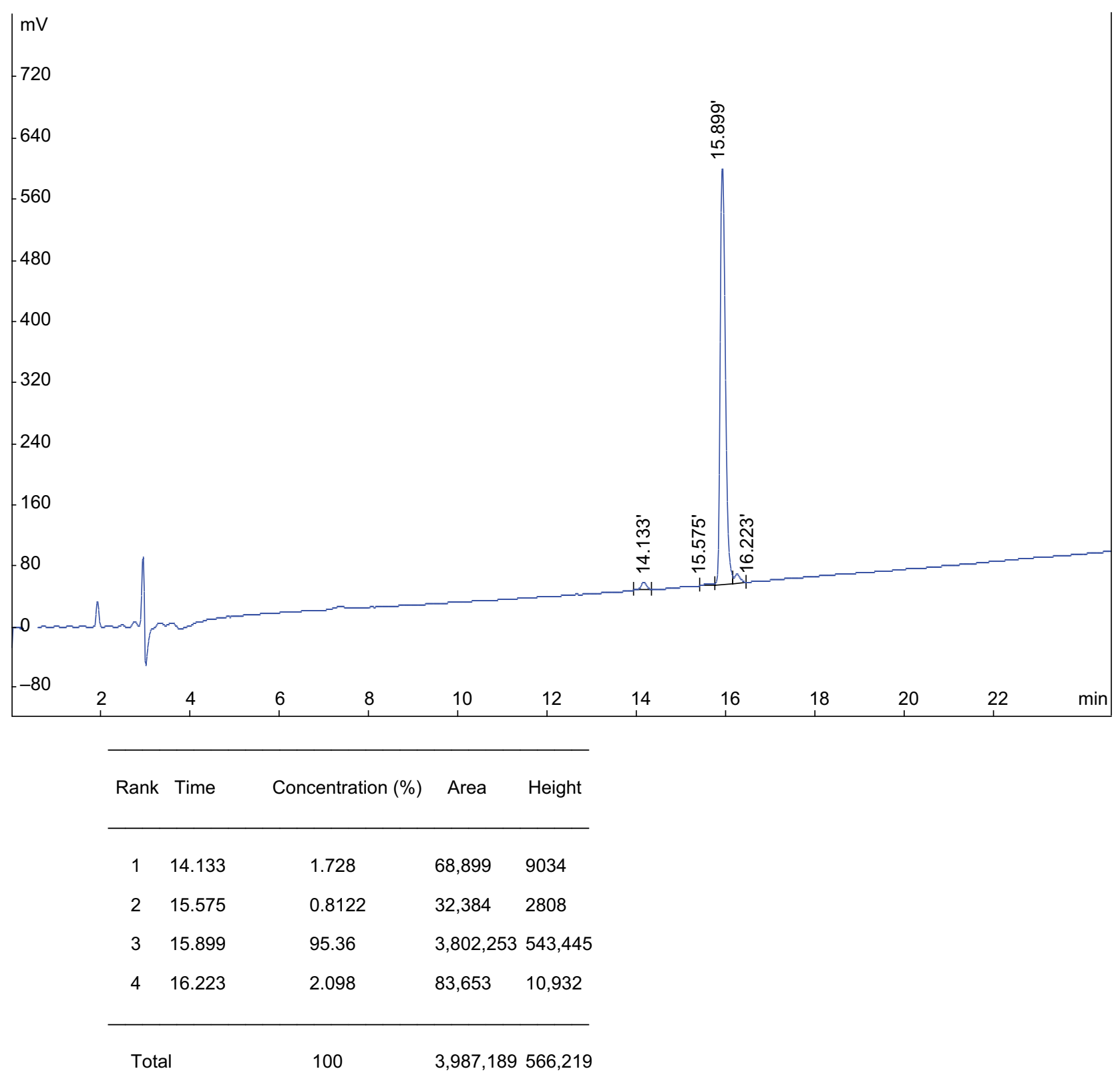

Figure I Analytical RP-HPLC chromatogram of the synthetic hybrid peptide $\mathrm{H} 4$ displaying a purity of $>95 \%$.

Abbreviation: RP-HPLC, reverse-phase high-performance liquid chromatography.

\section{Molecular modeling of $\mathrm{H} 4$}

The three-dimensional modeling of $\mathrm{H} 4$ revealed that the peptide is exhibiting an $\alpha$-helix conformation with the nearest template for reliable homology modeling was found with the KR-12 AMP and showed a score of 32.6 (Figure 3). The Ramachandran plot was used for model validation and confirmed that $100 \%$ of the amino acids participating in the formation of the secondary structure of the peptide are in favored regions in relation to the phi and psi torsion angles of the generated model. In addition, a generated z-score of
-0.68 was reported for $\mathrm{H} 4$ using the PROSA II software, which indicates that the generated model is of good quality.

\section{In vitro antimicrobial activity of $\mathrm{H} 4$}

The antimicrobial activity of the hybrid peptide $\mathrm{H} 4$ was evaluated against different representative strains (control and resistant strains) of Gram-positive and Gram-negative bacteria. $\mathrm{H} 4$ managed to inhibit the growth of all bacterial strains employed in this study (Table 3 ). The most sensitive Gram-positive organisms to H4's activity were the MDR 


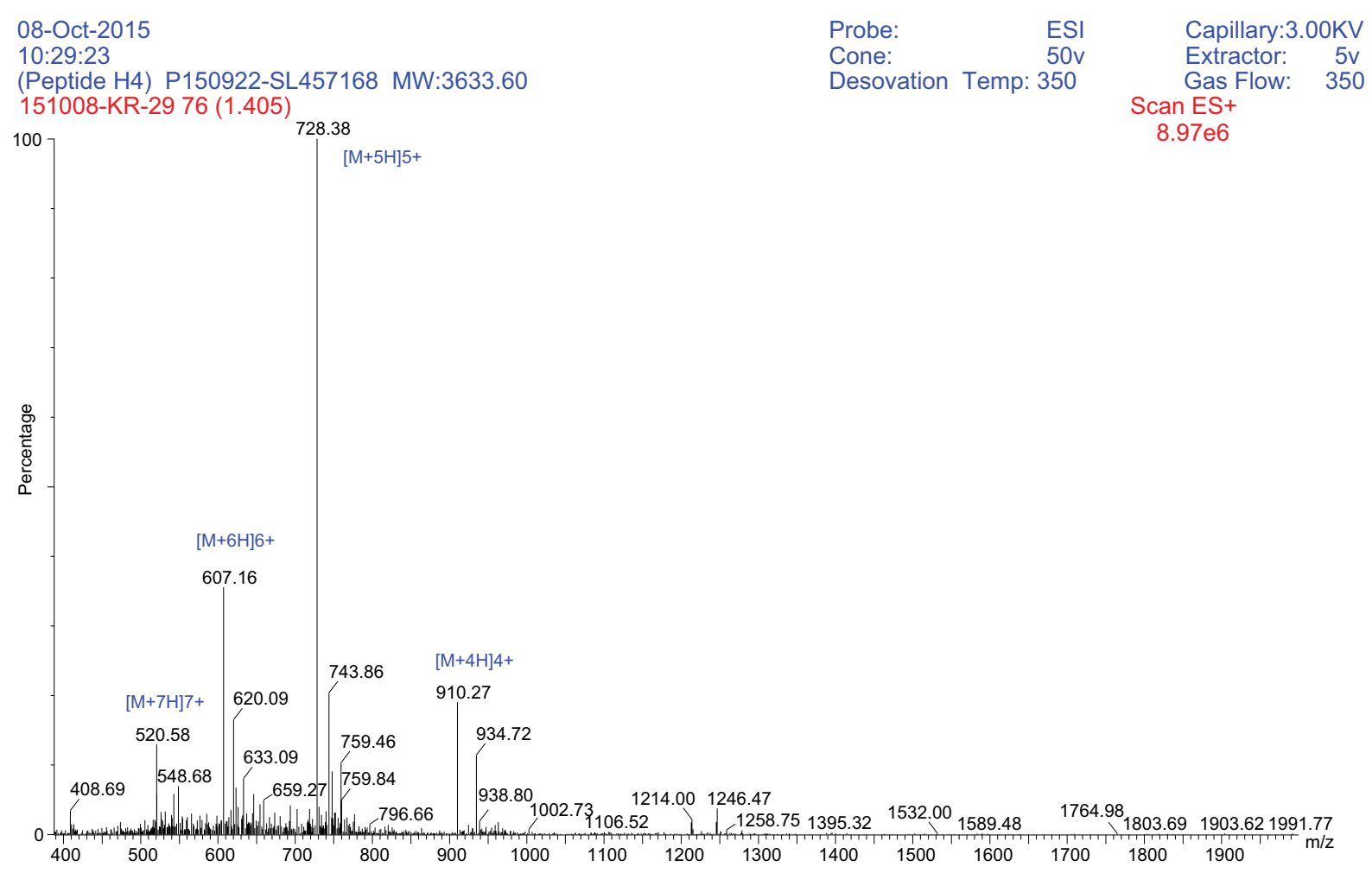

Figure 2 Positive ESI-MS analysis of the synthetic peptide H4 showing major peaks in the $+4,+5,+6$, and +7 charge states of $910.27,728.38,607.16$, and $520.68 \mathrm{Da}$, respectively.

Abbreviation: ESI-MS, electrospray ionization-mass spectrometry.

Table I NPS HNN secondary structure analysis and percentage helicity generated for BMAP-27, OP-I45, and the hybrid peptide H4 in addition to the $\alpha$-helical fragments identified from each peptide

\begin{tabular}{llll}
\hline Peptide & Natural sequence & Helicity $\%$ & a-helical fragments \\
\hline BMAP-27 & GRFKRFRKKFKKLFKKLSPVIPLLHL-NH2 & KFKKLFKKLSPV \\
& & & RFKRFRK \\
& & RFKRF \\
OP-145 & IGKEFKRIVERIKRFLRELVRPLR & GKEFKR \\
& & 91.8 & IGKEFKRI \\
H4 & KFKKLFKKLSPVIGKEFKRIVERIKRFLR & & IGKEFKRIVERIKRFL \\
& & 90 & KFKKLFKKLSPV \\
\hline
\end{tabular}

Abbreviation: NPS HNN, Hierarchical Neural Network in Network Protein Sequence.

strains of E. faecium and E. faecalis (ATCC BAA2316 and BAA2365) with an MIC value of $5 \mu \mathrm{M}$. The most resistant Gram-positive strain to H4's antimicrobial activity was S. aureus (ATCC 29213) with an MIC value of $20 \mu \mathrm{M}$, while the different strains of $S$. aureus including the control and MDR strains reported an MIC value of $10 \mu \mathrm{M}$ when exposed to H4. Regarding the antimicrobial activity of $\mathrm{H} 4$ against Gram-negative bacteria, the same potent activity of $\mathrm{H} 4$ was reported as the MIC values reported were in the range of 2.5-25 $\mu \mathrm{M}$. The most sensitive strain to H4's activity was the Gram-negative K. pneumoniae (ATCC 13883) with an MIC value of $2.5 \mu \mathrm{M}$, while the most resistant strain being the
MDR P. aeruginosa (ATCC BAA2114) strain with an MIC value of $25 \mu \mathrm{M}$. All the $\mathrm{MBC}$ values for all bacterial strains tested against $\mathrm{H} 4$ were equal to the MIC values, indicating that the peptide is behaving in a bactericidal manner since it is accepted that antimicrobial drugs that exhibit MBC values within the same range of MIC values and not more than fourfold are bactericidal.

\section{Antimicrobial synergistic assay}

In the present assay, $\mathrm{H} 4$ was combined with four conventional antibiotics (levofloxacin, chloramphenicol, rifampicin, and erythromycin), and the antimicrobial activity of these 
Table 2 The calculated mean hydrophobicity $<\mathrm{H}>$, hydrophobic moment $<\mu \mathrm{H}>$, and net charge of BMAP-27, OP-I45, and the hybrid peptide $\mathrm{H} 4$

\begin{tabular}{lllll}
\hline Peptide name & Hybrid sequence & Hydrophobicity $<$ H $>$ & Hydrophobic moment $<\boldsymbol{\mu H}>$ & Net charge z \\
\hline BMAP-27 & GRFKRFRKKFKKLFKKLSPVIPLLHL-NH2 & 0.394 & 0.474 & 10 \\
OP-I45 & GIGAVLKVLTTGLPALISWIKRKRQQ & 0.165 & 0.805 & 6 \\
H4 & KFKKLFKKLSPVIGKEFKRIVERIKRFLR & 0.272 & 0.638 & 6 \\
\hline
\end{tabular}

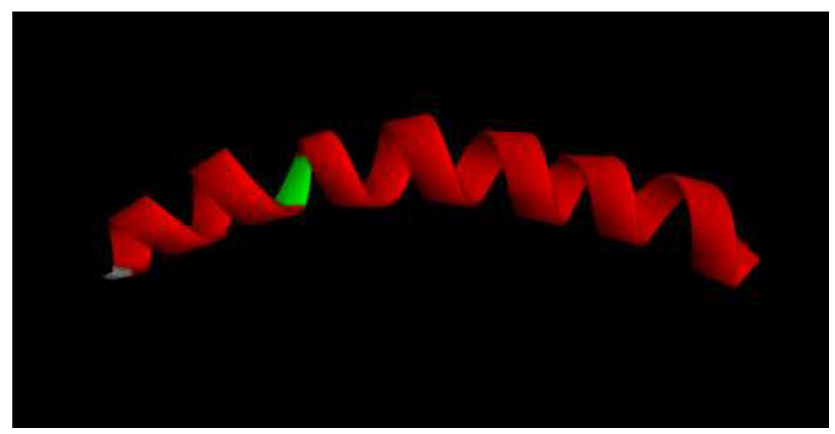

Figure 3 Three-dimensional structural modeling of $\mathrm{H} 4$. Red regions correspond to helical structures within the peptide, while the green regions represent hinged regions and unordered conformations, respectively. The structure was visualized using Accelrys Discovery Studio software.

Table 3 MICs of $\mathrm{H} 4$ against all the microbial strains employed in this study

\begin{tabular}{lll}
\hline Bacterial strain & ATCC & MIC $(\boldsymbol{\mu M})$ \\
\hline Standard strains (Gram-positive) & & \\
Staphylococcus aureus & 29213 & 20 \\
Enterococcus faecalis & 19433 & 15 \\
Staphylococcus epidermidis & 12228 & 10 \\
Resistant strains (Gram-positive) & & \\
S. aureus & 33591 & 10 \\
S. aureus & 43300 & 10 \\
S. aureus & BAA4I & 10 \\
E. faecalis & BAA2365 & 5 \\
Enterococcus faecium & BAA2316 & 5 \\
Standard strain (Gram-negative) & & \\
Escherichia coli & 25922 & 10 \\
Pseudomonas aeruginosa & 27853 & 20 \\
Acinetobacter baumannii & I9606 & 10 \\
Klebsiella pneumoniae & I3883 & 2.5 \\
Resistant strains (Gram-negative) & & \\
P. aeruginosa & BAA21 I4 \\
E. coli & BAA2452 & 25 \\
\hline
\end{tabular}

Abbreviations: ATCC, American Type Culture Collection; MIC, minimum inhibitory concentration.

combinations was assessed against five wild-type and MDR bacterial strains including E. faecalis (ATCC BAA2316), S. aureus (ATCC 29213 and 33591), and P. aeruginosa (ATCC BAA2114 and 27853).

\section{Determination of MIC values of the individual antibiotics}

The MIC values of the antibiotics employed in this study were determined against the tested bacterial strains. The reported data displayed wide variety of MIC values according to antibiotic and bacterial strains tested. E. faecalis (ATCC BAA2316) and S. aureus (ATCC 29213 and 33591) bacterial strains displayed high sensitivity toward rifampicin with MIC values equal to $7.5,0.4$, and $0.025 \mu \mathrm{M}$, respectively. Levofloxacin reported strong activity against $P$. aeruginosa (ATCC BAA2114 and 27853) bacterial strains with MIC values equal to 12 and $7.5 \mu \mathrm{M}$, respectively. On the other hand, chloramphenicol was reported as the most resistant antibiotic against the subjected bacterial strains, and the MIC values ranged from 20 to $350 \mu \mathrm{M}$. Table 4 summarizes all MIC values for the antibiotics.

\section{Checkerboard assay results}

The reported data, as shown in Table 4, displayed dramatic reduction in MIC values of $\mathrm{H} 4$ and the antibiotics combinations when compared to their individual MICs. Furthermore, the calculation of the percentage reduction of the MIC values of antibiotics in combination with $\mathrm{H} 4$ compared with MIC alone showed major differences. Of the overall 20 combinations, 12 combinations displayed synergistic behavior, while the last eight combinations showed an additive or an antagonistic effect. One of the most synergistic combinations was the H4-rifampicin combination that displayed a synergistic effect (FIC $\leq 0.5$ ) against all the subjected bacterial strains, and the percentage of reduction of the antibiotic MIC values ranged from $60 \%$ when tested against $P$. aeruginosa (ATCC BAA2114) to $80 \%$ when tested against $S$. aureus (ATCC 29213) in comparison with its individual MIC value. The H4-levofloxacin combination displayed synergistic effects against E. faecalis (ATCC 2316), S. aureus (ATCC 29213), and $P$. aeruginosa (ATCC 27853) with a percentage reduction of antibiotic MIC values compared with the individual MICs equal to $60 \%, 75 \%$, and $67 \%$, respectively. On the other hand, this combination displayed an additive effect ( $1 \geq$ FIC $>0.5)$ against $S$. aureus (ATCC 33591) and $P$. aeruginosa (ATCC BAA2114) with percentage MIC reductions equal to $67 \%$ and $33 \%$, respectively. Combining $\mathrm{H} 4$ with chloramphenicol led to the most synergistic effect in all combinations with a FIC value equal to 0.22 when tested against $P$. aeruginosa (ATCC BAA2114) and a percentage MIC reduction equal to $87 \%$. 
Table 4 MICs of individual antibiotics and those in combination with $\mathrm{H} 4$ including the percentage reduction of antibiotic MIC when in combination; MICs of individual $\mathrm{H} 4$ and those in antibiotic combination; and the FIC indices for the antimicrobial combinations against the tested bacterial species

\begin{tabular}{|c|c|c|c|c|c|c|c|}
\hline \multirow[t]{2}{*}{ Bacterial species } & \multicolumn{4}{|l|}{ Antibiotics } & \multicolumn{2}{|l|}{ H4 } & \multirow[t]{2}{*}{ FIC index } \\
\hline & & $\begin{array}{l}\text { Antibiotic } \\
\text { individual } \\
\text { MIC }(\mu \mathrm{M})\end{array}$ & $\begin{array}{l}\text { Antibiotic MIC } \\
\text { in combination } \\
(\mu \mathrm{M})\end{array}$ & $\begin{array}{l}\text { Reduction in } \\
\text { antibiotic } \\
\text { MIC (\%) }\end{array}$ & $\begin{array}{l}\mathrm{H} 4 \\
\text { individual } \\
\mathrm{MIC}(\mu \mathrm{M})\end{array}$ & $\begin{array}{l}\text { H4 MIC in } \\
\text { combination } \\
(\mu \mathrm{M})\end{array}$ & \\
\hline Enterococcus faecalis & Levofloxacin & 12.5 & 5 & 60 & 5 & 0.25 & 0.45 \\
\hline \multirow[t]{3}{*}{ ATCC BAA2316 } & Chloramphenicol & 20 & 15 & 25 & 5 & 0.25 & 0.8 \\
\hline & Rifampicin & 7.5 & 1.8 & 75 & 5 & 0.25 & 0.29 \\
\hline & Erythromycin & - & - & - & - & - & Antagonism \\
\hline Staphylococcus aureus & Levofloxacin & 0.75 & 0.25 & 67 & 10 & 7.5 & 1.08 \\
\hline \multirow[t]{3}{*}{ ATCC 3359I } & Chloramphenicol & 130 & 30 & 77 & 7.5 & 0.25 & 0.26 \\
\hline & Rifampicin & 0.4 & 0.1 & 75 & 7.5 & 0.2 & 0.27 \\
\hline & Erythromycin & - & - & - & - & - & Antagonism \\
\hline S. aureus ATCC & Levofloxacin & 0.5 & 0.125 & 75 & 20 & 2.5 & 0.37 \\
\hline \multirow[t]{3}{*}{29213} & Chloramphenicol & 20 & 10 & 50 & 20 & 5 & 0.75 \\
\hline & Rifampicin & 0.025 & 0.005 & 80 & 20 & 2.5 & 0.32 \\
\hline & Erythromycin & 0.5 & 0.125 & 75 & 20 & 2.5 & 0.37 \\
\hline Pseudomonas & Levofloxacin & 12 & 8 & 33 & 25 & 0.75 & 0.69 \\
\hline aeruginosa ATCC & Chloramphenicol & 200 & 25 & 87 & 25 & 2.5 & 0.22 \\
\hline \multirow[t]{2}{*}{ BAA2II4 } & Rifampicin & 50 & 20 & 60 & 25 & 2.5 & 0.5 \\
\hline & Erythromycin & - & - & - & - & - & Antagonism \\
\hline P. aeruginosa ATCC & Levofloxacin & 7.5 & 2.5 & 67 & 20 & 0.75 & 0.37 \\
\hline \multirow[t]{3}{*}{27853} & Chloramphenicol & 350 & 150 & 57 & 20 & 10 & 0.93 \\
\hline & Rifampicin & 45 & 15 & 67 & 20 & 0.25 & 0.34 \\
\hline & Erythromycin & 150 & 2.5 & 98 & 20 & 10 & 0.5 \\
\hline
\end{tabular}

Abbreviations: ATCC, American Type Culture Collection; FIC, fractional inhibitory concentration; MIC, minimum inhibitory concentration.

This combination also displayed another synergistic effect (FIC $=0.26$ ) when tested against $S$. aureus (ATCC 33591) and led to a percentage MIC reduction equal to $77 \%$. Testing H4-chloramphenicol combination against E. faecalis (ATCC 2316), S. aureus (ATCC 29213), and P aeruginosa (ATCC 27853) was accompanied with additive outcomes. Regarding H4-erythromycin combination, the synergistic effects were reported against $S$. aureus (ATCC 29213) and P. aeruginosa (ATCC 27853) with a percentage reduction of antibiotic MIC values equal to $75 \%$ and $98 \%$, respectively.

\section{Antibiofilm activity of $\mathrm{H} 4$}

Two representative resistant strains of both Gram-positive and Gram-negative bacteria including $S$. aureus (BAA41) and P. aeruginosa (BAA2114) were employed for the determination of the antibiofilm activity of $\mathrm{H} 4$. The MBEC for S. aureus (BAA41) and P. aeruginosa (BAA2114) was reported to be equal 20 and $25 \mu \mathrm{M}$, respectively (Table 5). The data obtained from the antibiofilm studies clearly indicated the potent antibiofilm activity of the $\mathrm{H} 4$ peptide at relatively low concentrations.
Table 5 Antibiofilm activity of $\mathrm{H} 4$ against standard resistant strains of Staphylococcus aureus (BAA4I) and Pseudomonas aeruginosa (BAA2। I4)

\begin{tabular}{lll}
\hline $\begin{array}{l}\text { Antibiofilm } \\
\text { activity }\end{array}$ & $\begin{array}{l}\text { S. aureus } \\
\text { (ATCC BAA4I) }\end{array}$ & $\begin{array}{l}\text { P. aeruginosa } \\
\text { (BAA2 I I4) }\end{array}$ \\
\hline MBEC value & $20 \mu \mathrm{M}$ & $25 \mu \mathrm{M}$ \\
\hline
\end{tabular}

Abbreviations: ATCC, American Type Culture Collection; MBEC, minimum biofilm eradication concentration.

\section{Hemolytic assay}

The hemolytic activity of $\mathrm{H} 4$ against human erythrocytes was evaluated at different concentrations within the range of 5-100 $\mu \mathrm{M}$. At the antimicrobial concentrations required to inhibit the growth of both Gram-positive and Gram-negative microbial strains evaluated in this study which range between 2.5 and $25 \mu \mathrm{M}, \mathrm{H} 4$ caused $0 \%$ hemolysis after 1 hour of incubation with human erythrocytes (Table 6). In addition, when $\mathrm{H} 4$ was incubated with human erythrocytes at concentrations equal to the MBEC values $(25 \mu \mathrm{M})$ that are needed to inhibit biofilm growth of $P$. aeruginosa and $S$. aureus, respectively, the peptide as with the MIC values induced $0 \%$ hemolysis. When evaluated at a concentration equivalent to tenfold the 
mean MIC $(100 \mu \mathrm{M})$, the percentage hemolysis reported was equal to 2.1 after 1 hour of incubation with human erythrocytes. The hemolytic results clearly indicated the peptide's significant selectivity toward microbial cells when compared to human erythrocytes, confirming the rationale behind decreasing the toxicity of AMPs as a result of the hybridization strategy employed in this study.

\section{Cell cytotoxicity of $\mathrm{H} 4$}

H4 managed to inhibit the proliferation of both mammalian cell lines HEK293 and Vero that were employed in this study with average half maximal inhibitory concentration $\left(\mathrm{IC}_{50}\right)$

Table 6 Hemolytic activity of $\mathrm{H} 4$ against human erythrocytes after I hour of exposure to the peptide

\begin{tabular}{ll}
\hline Peptide concentration $(\boldsymbol{\mu M})$ & Hemolysis $(\%) \mathbf{H 4}$ \\
\hline 5 & 0 \\
10 & 0 \\
25 & 0 \\
40 & 0 \\
55 & 0 \\
70 & 0 \\
85 & 0 \\
100 & 2.1 \\
\hline
\end{tabular}

values of 61.9 and $64.9 \mu \mathrm{M}$, respectively (Figure 4; Table 7). When compared with the data obtained from the antimicrobial susceptibility assay, a sixfold increase in the values of the geometric MIC was needed to inhibit 50\% proliferation of mammalian cells when treated with $\mathrm{H} 4$ peptide. The results of the antiproliferative study and the previous hemolytic studies indicated that the peptide is exhibiting minimal cytotoxicity against mammalian cells when compared to its antimicrobial activity. In addition, the peptide is showing a significant margin of selectivity against microbial cells and thus confirming the benefit of both hybridization and synergism in decreasing the overall cytotoxicity of AMPs.

\section{Discussion}

The bacterial resistance issue has become one of the most threatening and challenging health dilemmas in the recent era. Globally, this serious threat is no longer a prediction

Table 7 Cytotoxicity effects $\left(\mathrm{IC}_{50}\right.$ value) using MTT assay of $\mathrm{H} 4$ on two eukaryotic mammalian cell lines (HEK293 and Vero)

\begin{tabular}{lll}
\hline Peptide & HEK293 IC $_{50}(\mu \mathrm{M})$ & Vero IC $(\mu \mathrm{M})$ \\
\hline $\mathrm{H} 4$ & $61.9 \pm 0.18$ & $64.9 \pm 1.0 \mathrm{I}$ \\
\hline
\end{tabular}

Note: Data represent the mean \pm SD of three independent experiments. Abbreviations: $\quad I_{50}$, half maximal inhibitory concentration; MTT, methylthiazolyldiphenyl-tetrazolium bromide.

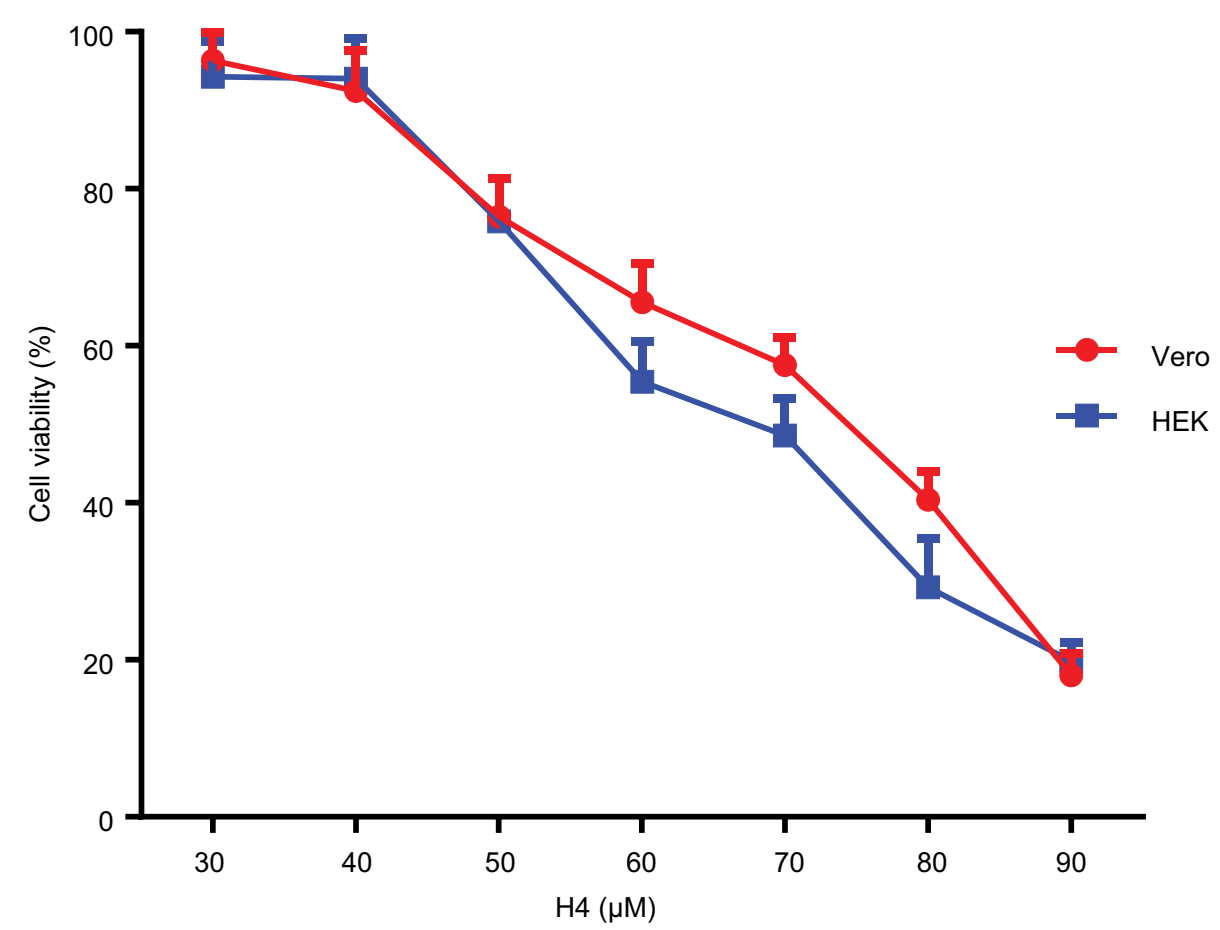

Figure 4 Cell survival curves as measured by MTT assay for $\mathrm{H} 4$ against two normal mammalian cell lines, Vero and HEK293. Cells were incubated with various concentrations of the peptides, for 24 hours, at $37^{\circ} \mathrm{C}$. Control cells represent $100 \%$ proliferation, and the mean absorbance of treated cells was related to control values to determine sensitivity. Error bars represent standard error from mean cell proliferation as determined by repeated experiments.

Abbreviation: MTT, methylthiazolyldiphenyl-tetrazolium bromide. 
for the future; it is happening right now in every region of the world and has the potential to affect anyone, of any age, in any country. ${ }^{25}$ This state of uncontrolled and progressive acceleration of microbial resistance, including the MDRB, has been further complicated due to the significant decline in the available effective antibiotics within the health care setting. The last few decades have witnessed drying pipelines within the major pharmaceutical companies and reduced budgets allocated for research and development in the field of antimicrobials. ${ }^{26-29}$ Thus, recent efforts have been directed to develop new antimicrobial agents with new modes of action. AMPs exemplify a promising group of therapeutics to manage bacterial infections including MDRB. The ability to kill bacterial cells rapidly and the very low likelihood of resistance development represent considerable advantages over traditional antibiotics. ${ }^{30}$ In contrast, many disadvantages restrict the use of native peptides as therapeutics. The AMPs' toxicity against mammalian cells is considered a major hindrance to the development of effective antimicrobial agents. ${ }^{31}$ In spite of all these negative aspects, AMPs still attract a growing interest and are considered as effective tools to develop new therapeutics. Peptidomimetics are a potential strategy that has recently been followed to enhance the antimicrobial activity and bioavailability of native AMPs. This approach is based on maintaining biological activity via identifying active fragments with different peptide sequences instead of introducing specific structural and functional physicochemical modifications. ${ }^{32}$ In addition, recent studies revealed that peptidomimetics may also enhance high cell selectivity toward mammalian cells and enzymatic resistance over native peptides. ${ }^{33,34}$ Hybridization, a well-representative strategy of peptidomimetics, is an effective trend aimed at enhancing the selectivity, diminishing toxicity, and potentiating or maintaining the antimicrobial activity of AMPs. ${ }^{33}$ For effective deployment of the hybridization strategy, several physicochemical parameters should be taken in consideration that mainly influence AMPs' antimicrobial activity and differential selectivity. In the present study, individual $\alpha$-helical fragments from both BMAP-27 and OP-145 were merged rationally to design $\mathrm{H} 4$, a novel hybrid peptide that exhibits an enhanced therapeutic index profile while retaining the antimicrobial activity of parent native peptides. The secondary structure, hydrophobicity, and net charge are considered the most important factors that govern AMPs' activity. ${ }^{36}$ Thus, the newly designed hybrid peptide was screened for all the structural parameters including the percentage of helicity, net charge, hydrophobicity, and hydrophobic moment that give a primary indication about its antimicrobial activity.
In order to elicit their antimicrobial activity, AMPs and bacterial cell membranes should first accomplish an initial binding step. This issue is mainly governed by the electrostatic attraction between the negatively charged surfaces of bacterial cell membranes and hydrophilic positively charged residues of AMPs. This facilitates the insertion of the hydrophobic motifs of AMPs into the bacterial membrane, leading to destabilization of bacterial cell membrane and hence cell lysis and death as a result of transient pore-like structure formation. ${ }^{37}$ In addition, maintaining a threshold degree of hydrophobicity is crucial to disrupt bacterial membrane integrity after membrane penetration. ${ }^{38}$

As reported in the present study, $\mathrm{H} 4$ exhibits a net cationic charge equivalent to +6 . This significant charge load ensures a sufficient electrostatic attraction between the cationic residues of $\mathrm{H} 4$ and the negatively charged phospholipids found on bacterial cell membranes. ${ }^{39}$ The presence of arginine residues within the $\mathrm{H} 4$ sequence, especially on the terminal region of the peptide, has a vital role for potentiating an electrostatic attraction by creating a complex between arginine's guanidine moiety and the phospholipid head groups of bacterial cell membranes. ${ }^{40}$ In addition, the secondary structure is considered as a detrimental factor that also potentiates AMPs' permeation and hence enhances the killing potency against bacterial cells. Thus, the design strategy of $\mathrm{H} 4$, a novel hybrid peptide, depends on combining two active $\alpha$-helical fragments identified in the parent native peptides employed in this study. This hybridization strategy aims at improving the characteristics of the newly generated peptide over the parent peptides via merging and capturing the potential benefits of each individual $\alpha$-helical fragment. ${ }^{41}$ According to the helical analysis data shown in Table 1, each individual parent peptide seems to exhibit three potential $\alpha$-helical fragments suitable for hybridization. In the initial screening assay, H4 is the only one, of the five newly generated hybrid peptides that displayed promising antimicrobial activity. The data and the selected fragments of BMAP-27 that constitute the primary sequence of $\mathrm{H} 4$ are also in accordance with previous studies performed on BMAP-27 and its active N- and $\mathrm{C}$-terminal parts that clearly indicate the importance of the amino acids incorporated in the primary structure on reducing the cytotoxicity of the peptide. ${ }^{42}$

Accordingly, when $\mathrm{H} 4$ was assessed against representative wild-type and MDR bacterial strains, the results revealed that the new rationally designed hybrid peptide displays a wide spectrum of antimicrobial activity against all the employed bacterial strains. The MIC values of $\mathrm{H} 4$ against the representative standard strains were within the range of 
$10-20 \mu \mathrm{M}$ and decreased to $5-10 \mu \mathrm{M}$ when tested against the representative resistant strains of Gram-positive bacteria. On the other hand, H4 managed to kill the representative standard as well as MDR bacterial strains of Gram-negative bacteria within the range of 10-25 $\mu \mathrm{M}$, except for K. pneumoniae, that was the most sensitive strain with an MIC value equivalent to $2.5 \mu \mathrm{M}$. Therefore, the resistant nature of the subjected bacterial strains seems to have no effects on the mechanism of action of the AMP and hence its killing potential. All the reported MIC values were equal to the MBC values, indicating that the $\mathrm{H} 4$ peptide is bactericidal in nature rather than bacteriostatic. All these results prove the efficiency of the hybridization strategy in developing highly active and promising antimicrobial agents.

Furthermore, combining $\mathrm{H} 4$ peptide with different conventional antibiotics and assessing the antimicrobial and synergistic activity of these combinations, $60 \%$ of the resulted combinations displayed a synergistic behavior, while only $15 \%$ displayed an antagonistic effect. This high diversity and variation in the MIC results within the different antibiotic-H4 combinations can be explained by the high diversity in the mechanism of actions of the employed antibiotics alone as well as in combination with $\mathrm{H} 4$. As the bacterial cell membrane is suggested to be the target of $\mathrm{H} 4$, the subjected antibiotics act intracellularly by interfering with nucleic acid synthesis or blocking different protein synthesis pathways. ${ }^{43,44}$ However, there is no explanation to the synergistic mechanism of action of $\mathrm{H} 4$-antibiotic combinations. One of the most accepted theories is that membrane targeting and pore formation effects of $\mathrm{H} 4$ could facilitate antibiotic insertion through bacterial cell membrane and consequently lead to facilitating the antibiotic mechanism of action intracellularly. ${ }^{45}$

The biofilm structure is considered as one of the most resistant mechanisms of bacterial cell survival that cannot be managed by the available conventional antibiotics. Usually and in comparison with planktonic cells, it takes tenfold to 1000-fold higher concentrations of traditional antibiotics to eradicate biofilm cells due to their specific nature of resistance expression, including the extracellular lipopolysaccharide matrix that retards antibiotic penetration and hence leads to the loss of treatment efficiency. ${ }^{46,47}$ However, when H4 was evaluated for its antibiofilm activity, the results revealed that $\mathrm{H} 4$ has a potential ability to eradicate biofilm formation as its reported $\mathrm{MBEC}$ values were equivalent to the $\mathrm{MIC}$ values of both $S$. aureus (ATCC BAA41) and P. aeruginosa (ATCC BAA2114), respectively. The results are a good indication that $\mathrm{H} 4$ is a promising potent antibiofilm agent; however, the mechanism of action of $\mathrm{H} 4$ against biofilm structures remains to be elucidated.

The benefits of the hybridization strategy are also highlighted in this study via evaluating the cytotoxicity profiles of the newly designed hybrid peptide against human erythrocytes and two mammalian cell lines. This toxicity is almost diminished as the new hybrid peptide did not exhibit any hemolytic toxicity at the concentrations required to kill or inhibit biofilm and planktonic bacterial cells, and the percentage of hemolysis never exceeded 2.1 when exposed to a tenfold increase in concentration compared with the average $\mathrm{MIC}$ values reported against all studied bacterial strains. The cytotoxic nature associated with the parent peptide, BMAP27 , against normal mammalian cells is also minimal as the reported $\mathrm{IC}_{50}$ values for $\mathrm{H} 4$ against both employed mammalian cell lines were equivalent to six-fold the average MIC values of the antimicrobial susceptibility assay. Accordingly, the reported data confirmed that the hybridization strategy via merging two individual $\alpha$-helical fragments of both parent peptides is an effective way to enhance the selectivity and potentially diminish the toxicity of AMPs against eukaryotic cells. The low hydrophobicity (0.272) and significant positive charge (+6) of $\mathrm{H} 4$ contribute to minimizing the cytotoxicity of the peptide toward eukaryotic cells. The abundant presence of zwitterionic phospholipids in eukaryotic cells in contrast to bacterial cell membranes that carry a higher proportion of anionic phospholipids in their membrane structure plays a major role in enhancing the selectivity of positively charged AMPs. ${ }^{48,49}$ The most significant results of this study are related to the synergistic assay. As the aim of this study was to reduce the cytotoxicity of AMPs, the synergistic studies of H4 with conventional antibiotics have reduced the effective MICs of both $\mathrm{H} 4$ and the antibiotics dramatically, and the lowest FIC index was reported for the chloramphenicol-H4 combination against the resistant Gram-negative $P$. aeruginosa (ATCC BAA2114) with a FIC value of 0.22. The MIC value for $\mathrm{H} 4$ in that combination was reduced tenfold from 25 to $2.5 \mu \mathrm{M}$, while chloramphenicol's MIC was reduced from 200 to $25 \mu \mathrm{M}$. The same combination was also proved to be effective against $S$. aureus (ATCC 43300) as the MIC value for $\mathrm{H} 4$ decreased 20 -fold from 5 to $0.25 \mu \mathrm{M}$. In addition, other $\mathrm{H} 4$ antibiotic combinations were also proved to be successful such as the rifampicin-H4 combination that displayed a synergistic mode of action against all bacterial strains tested. The results of the synergistic studies indicated that synergism is effective in reducing AMPs' MIC values effectively and consequently can reduce the toxicity of AMPs significantly. Although the mechanism of action of the synergistic mode 
of action remains to be elucidated, our study has proved that both hybridization and synergism are effective tools in reducing the overall cytotoxicity of AMPs and that several synergistic AMP-antibiotic combinations can be identified for novel antimicrobial development.

\section{Conclusion}

The present study reported the design of a novel hybrid AMP named $\mathrm{H} 4$ via merging two individual $\alpha$-helical fragments from two predefined native parent peptides. Evaluating the hemolytic and antiproliferative activity of $\mathrm{H} 4$ revealed the advanced beneficial effects of the hybridization as the newly generated peptide exhibited very low or negligible toxicity profiles toward eukaryotic cells. Furthermore, its antimicrobial activity and toxicity profiles were dramatically enhanced when combined with conventional antibiotics, and synergistic modes of action were the dominant trend between the peptide-antibiotic combinations. Further investigations are required using animal models in order to optimize H4's pharmacodynamic and pharmacokinetic profiles in order to assess the feasibility of employing the molecule into advanced trials as an efficient and nontoxic antimicrobial agent.

\section{Acknowledgments}

This study was generously supported by the Jordanian Scientific Research Fund (grant number: MPH/1/15/2014). The authors would also like to thank the Deanship of Research at Jordan University of Science and Technology for their logistical and administrative support of this project.

\section{Disclosure}

The authors report no conflicts of interest in this work.

\section{References}

1. Roca I, Akova M, Baquero F, et al. The global threat of antimicrobial resistance: science for intervention. New Microbes New Infect. 2015;6:22-29.

2. Luepke KH, Suda KJ, Boucher H, et al. Past, present, and future of antibacterial economics: increasing bacterial resistance, limited antibiotic pipeline, and societal implications. Pharmacotherapy. 2017;37(1):71-84.

3. Spellberg B. The future of antibiotics. Crit Care. 2014;18(3):228.

4. Kang SJ, Park SJ, Mishig-Ochir T, Lee BJ. Antimicrobial peptides: therapeutic potentials. Expert Rev Anti Infect Ther. 2014;12(12): 1477-1486.

5. Sun E, Belanger CR, Haney EF, Hancock REW. Host defense (antimicrobial) peptides. In: Peptide Applications in Biomedicine, Biotechnology and Bioengineering. Woodhead Publishing. 2018:253-285.

6. Strempel N, Strehmel J, Overhage J. Potential application of antimicrobial peptides in the treatment of bacterial biofilm infections. Curr Pharm Des. 2015;21(1):67-84.
7. Mahlapuu M, Håkansson J, Ringstad L, Björn C. Antimicrobial peptides: an emerging category of therapeutic agents. Front Cell Infect Microbiol. 2016;6:194.

8. Pompilio A, Scocchi M, Pomponio S, et al. Antibacterial and anti-biofilm effects of cathelicidin peptides against pathogens isolated from cystic fibrosis patients. Peptides. 2011;32(9):1807-1814.

9. Malanovic N, Leber R, Schmuck M, et al. Phospholipid-driven differences determine the action of the synthetic antimicrobial peptide OP-145 on Gram-positive bacterial and mammalian membrane model systems. Biochim Biophys Acta. 2015;1848(10):2437-2447.

10. Peters BM, Shirtliff ME, Jabra-Rizk MA. Antimicrobial peptides: primeval molecules or future drugs? PLoS Pathog. 2010;6(10):e1001067.

11. Algburi A, Zhang Y, Weeks R, et al. Gemini cationic amphiphiles control biofilm formation by bacterial vaginosis pathogens. Antimicrob Agents Chemother. 2017;61(12):e00650-e00717.

12. Srivastava $\mathrm{S}$, Ghosh JK. Introduction of a lysine residue promotes aggregation of temporin $\mathrm{L}$ in lipopolysaccharides and augmentation of its antiendotoxin property. Antimicrob Agents Chemother. 2013;57(6):2457-2466.

13. Song R, Shi Q, Yang P, Wei R. Identification of antibacterial peptides from Maillard reaction products of half-fin anchovy hydrolysates/glucose via LC-ESI-QTOF-MS analysis. J Funct Foods. 2017;36:387-395.

14. Faccone D, Veliz O, Corso A, et al. Antimicrobial activity of de novo designed cationic peptides against multi-resistant clinical isolates. Eur J Med Chem. 2014;71:31-35.

15. Lear S, Cobb SL. Pep-Calc.com: a set of web utilities for the calculation of peptide and peptoid properties and automatic masss spectral peak assignment. J Comput Aided Mol Des. 2016;30(3):271-277.

16. Gasteiger E, Gattiker A, Hoogland C, Ivanyi I, Appel RD, Bairoch A. ExPASy: the proteomics server for in-depth protein knowledge and analysis. Nucleic Acids Res. 2003;31(13):3784-3788.

17. Söding J, Biegert A, Lupas AN. The HHpred interactive server for protein homology detection and structure prediction. Nucleic Acids Res. 2005;33(Web Server Issue):W244-W248.

18. Šali A, Blundell TL. Comparative protein modelling by satisfaction of spatial restraints. J Mol Bio. 1993;234(3):779-815.

19. Almaaytah A, Tarazi S, Abu-Alhaijaa A, et al. Enhanced antimicrobial activity of AamAP1-Lysine, a novel synthetic peptide analog derived from the scorpion venom peptide AamAP1. Pharmaceuticals (Basel). 2014;7(5):502-516.

20. Ouhara K, Komatsuzawa H, Kawai T, et al. Increased resistance to cationic antimicrobial peptide LL-37 in methicillin-resistant strains of Staphylococcus aureus. JAntimicrob Chemother. 2008;61(6):1266-1269.

21. Almaaytah A, Qaoud MT, Khalil Mohammed G, et al. Antimicrobial and antibiofilm activity of UP-5, an ultrashort antimicrobial peptide designed using only arginine and biphenylalanine. Pharmaceuticals (Basel). 2018;11(1):pii:E3.

22. Sueke H, Kaye SB, Neal T, Hall A, Tuft S, Parry CM. An in vitro investigation of synergy or antagonism between antimicrobial combinations against isolates from bacterial keratitis. Invest Ophthalmol Vis Sci. 2010;51(8):4151-4155.

23. Wang W, Tao R, Tong Z, et al. Effect of a novel antimicrobial peptide chrysophsin-1 on oral pathogens and Streptococcus mutans biofilms. Peptides. 2012;33(2):212-219.

24. Almaaytah A, Tarazi S, Alsheyab F, Al-Balas Q, Mukattash T. Antimicrobial and antibiofilm activity of mauriporin, a multifunctional scorpion venom peptide. Int J Pept Res Ther. 2014;20(4):397-408.

25. World Health Organization. Antimicrobial Resistance. Geneva: World Health Organization. Available from: http://www.who.int/mediacentre/ factsheets/fs194/en/. Accessed November 13, 2017.

26. Schäberle TF, Hack IM. Overcoming the current deadlock in antibiotic research. Trends Microbiol. 2014;22(4):165-167.

27. Davies J, Davies D. Origins and evolution of antibiotic resistance. Microbiol Mol Biol Rev. 2010;74(3:417-433.

28. Leung E, Weil DE, Raviglione M, Nakatani H. The WHO policy package to combat antimicrobial resistance. Bull World Health Organ. 2011;89(5):390-392. 
29. Rolain JM, Canton R, Cornaglia G. Emergence of antibiotic resistance: need for a new paradigm. Clin Microbiol Infect. 2012;18(7): 615-616.

30. Mataraci E, Dosler S. In vitro activities of antibiotics and antimicrobial cationic peptides alone and in combination against methicillin-resistant Staphylococcus aureus biofilms. Antimicrob Agents Chemother. 2012;56(12): 6366-6371.

31. Marr AK, Gooderham WJ, and Hancock RE. Antibacterial peptides for therapeutic use: obstacles and realistic outlook. Curr Opin Pharmacol. 2006;6(5):468-472.

32. Trabocchi A, Guarna A. The basics of peptidomimetics. In: Peptidomimetics in Organic and Medicinal Chemistry: The Art of Transforming Peptides in Drugs. John Wiley \& Sons, Ltd. 2014:1-17.

33. Liskamp RMJ. Conformationally restricted amino acids and dipeptides, (non)peptidomimetics and secondary structure mimetics. Recueil des Travaux Chimiques des Pays-Bas. 1994;113(1):1-19.

34. Olson GL, Bolin DR, Bonner MP, et al. Concepts and progress in the development of peptide mimetics. J Med Chem. 1993;36(21): 3039-3049.

35. Almaaytah A, Tarazi S, Al-Fandi M, Abuilhaija A, Al-Balas Q, AbuAwad A. The design and functional characterization of the antimicrobial and antibiofilm activities of BMAP27-melittin, a rationally designed hybrid peptide. Int J Pept Res Ther. 2015;21(2):165-177.

36. Huang Y, Huang J, Chen Y. Alpha-helical cationic antimicrobial peptides: relationships of structure and function. Protein Cell. 2010;1(2):143-152.

36. Pasupuleti M, Schmidtchen A, Malmsten M. Antimicrobial peptides: key components of the innate immune system. Crit Rev Biotechnol. 2012;32(2):143-171.

38. Fjell CD, Hiss JA, Hancock RE, Schneider G. Designing antimicrobial peptides: form follows function. Nat Rev Drug Discov. 2011;11(1):37-51.

39. Lau QY, Ng FM, Cheong JW, et al. Discovery of an ultra-short linear antibacterial tetrapeptide with anti-MRSA activity from a structureactivity relationship study. Eur J Med Chem. 2015;105:138-144.

40. Wang G. Determination of solution structure and lipid micelle location of an engineered membrane peptide by using one NMR experiment and one sample. Biochim Biophys Acta. 2007;1768(12):3271-3281.
41. Huang Y, He L, Li G, Zhai N, Jiang H, Chen Y. Role of helicity of $\alpha$-helical antimicrobial peptides to improve specificity. Protein Cell. 2014;5(8):631-642.

42. Ahmad A, Azmi S, Srivastava RM, et al. Design of nontoxic analogues of cathelicidin-derived bovine antimicrobial peptide BMAP-27: the role of leucine as well as phenylalanine zipper sequences in determining its toxicity. Biochemistry. 2009;48(46):10905-10917.

43. Ruden S, Hilpert K, Berditsch M, Wadhwani P, Ulrich AS. Synergistic interaction between silver nanoparticles and membrane-permeabilizing antimicrobial peptides. Antimicrob Agents Chemotherapy. 2009;53(8):3538-3540.

44. Maróti G, Kereszt A, Kondorosi E, Mergaert P. Natural roles of antimicrobial peptides in microbes, plants and animals. Res Microbiol. 2011;162(4):363-374.

45. Yang P, Ramamoorthy A, Chen Z. Membrane orientation of MSI-78 measured by sum frequency generation vibrational spectroscopy. Langmuir. 2011;27(12):7760-7767.

46. Zhang Y, Liu Y, Sun Y, et al. In vitro synergistic activities of antimicrobial peptide brevinin-2CE with five kinds of antibiotics against multidrug-resistant clinical isolates. Curr Microbiol. 2014;68(6):685-692.

47. Hirt H, Gorr SU. Antimicrobial peptide GL13K is effective in reducing biofilms of Pseudomonas aeruginosa. Antimicrob Agents Chemother. 2013;57(10):4903-4910.

48. Mishra B, Wang G. Individual and combined effects of engineered peptides and antibiotics on Pseudomonas aeruginosa biofilms. Pharmaceuticals (Basel). 2017;10(3):pii:E58.

49. Høiby N, Ciofu O, Johansen HK, et al. The clinical impact of bacterial biofilms. Int J Oral Sci. 2011;3(2):55-65.

50. Olson ME, Ceri H, Morck DW, Buret AG, Read RR. Biofilm bacteria: formation and comparative susceptibility to antibiotics. Can JVet Res. 2002;66(2):86-92.

51. Mohandas N, Gallagher PG. Red cell membrane: past, present, and future. Blood. 2008;112(10):3939-3948.

52. Glukhov E, Stark M, Burrows LL, Deber CM. Basis for selectivity of cationic antimicrobial peptides for bacterial versus mammalian membranes. J Biol Chem. 2005;280(40):33960-33967.
Infection and Drug Resistance

\section{Publish your work in this journal}

Infection and Drug Resistance is an international, peer-reviewed openaccess journal that focuses on the optimal treatment of infection (bacterial, fungal and viral) and the development and institution of preventive strategies to minimize the development and spread of resistance. The journal is specifically concerned with the epidemiology of antibiotic

\section{Dovepress}

resistance and the mechanisms of resistance development and diffusion in both hospitals and the community. The manuscript management system is completely online and includes a very quick and fair peerreview system, which is all easy to use. Visit http://www.dovepress.com/ testimonials.php to read real quotes from published authors. 